\title{
Hammocks in the Neonatal ICU: Still a Long Way From Vacation
}

Hammocks have been used in many cultures, including among Brazilian Indians, since the 1500s, as recorded by a letter sent by Pedro Vaz deCaminha to the king of Portugal. ${ }^{1}$ Although its use has declined since then, it is still used in a few areas in Central and South America. ${ }^{2}$ Moreover, in past decades, with increased commercial availability of a variety of small hammocks, the hammock has gained more acceptance and use, especially in the care of younger infants. This increased utilization has led to a few case series that reported advantages of its use in both term and preterm neonates, with improvement in vital signs, decrease in stress, better weight gain, and improved shortterm neuromuscular maturity. ${ }^{3}$ These studies were limited by a lack of long-term follow-up and speculated that the decrease in stress and better weight gain during a neonatal ICU stay may be associated with better long-term neuropsychomotor growth. ${ }^{4}$

Ribas et $\mathrm{al}^{5}$ performed a pilot study on the use of hammocks in preterm neonates in the neonatal ICU, with improvement in pain scores and the sleep-wake cycle. The strength of this study, compared with the previous case series, was in the careful selection of its study population, which consisted of preterm neonates who were at $>30$ week of gestational age and clinically stable when placed in the hammock. Placing preterm neonates ages $<30$ week in the hammock could cause positioning difficulties, such as flexion of neck. This abnormal positioning may cause repeated desaturation that has been linked to poor developmental outcomes.

By selecting those $>30$-week-old stable neonates, $\mathrm{Ri}$ bas et $\mathrm{al}^{5}$ highlighted the importance of the appropriate selection of neonates for hammock use, based on their gestational age and postnatal age, to optimize the benefits to be gained from the use of a hammock while minimizing the risks and unwanted effects, including desaturations. Furthermore, the authors studied the impact of hammock use on both pain and the sleep-wake cycle among the neonates ${ }^{5}$ both of which are important independent surrogate markers for short- and long-term neurodevelopmental

\footnotetext{
The author has disclosed no conflicts of interest.
}

Correspondence: Shantanu Rastogi MD MMM, SUNY-HSC at Brooklyn, Maimonides Infant and Children's Hospital, 4802 Tenth Ave, F-119, Brooklyn NY 11219. E-mail: srastogi@maimonidesmed.org.

DOI: $10.4187 /$ respcare. 06983 outcomes in preterm neonates. However, in keeping with the earlier case studies, ${ }^{3,4}$ one of the limitations of the study by Ribas et $\mathrm{al}^{5}$ was the lack of long-term follow-up, which remains an area that needs further investigation.

See the Original Study on Page 384

Based on the findings of this study, there are some methodological and clinical aspects that need to be addressed in future studies. There are several improved methods for monitoring vital signs, and one such approach is the use of continuous monitoring to reduce the noise and natural variability in the recording. 6 Further, positioning of the baby in the hammock needs careful attention. The authors compared the lateral position both for nesting and in the hammock ${ }^{5}$; also, there are precautions required when placing the baby in the lateral position. There is a risk of the cloth covering the face of the baby while the baby is in the hammock, which may lead to frequent desaturations, and, if the parents continue this practice at home, there is a risk of death. ${ }^{7}$

Furthermore, the American Academy of Pediatrics, in its policy on sudden infant death syndrome and other sleeprelated infant deaths, specifically recommends the supine sleeping position, except for few rare exceptions in which upper-airway protective mechanisms may be impaired. ${ }^{8} \mathrm{~A}$ firm sleep surface and not using slings and swings are additional safe sleeping recommendations by the American Academy of Pediatrics. ${ }^{8}$ These recommendations are at odds with the use of a hammock for neonates and thus may miss the developmental advantages that hammock use might have to offer.

By balancing the correct patient selection, appropriate window of postnatal age, and the duration of hammock use in the neonatal ICU, future studies can determine which neonates would get the best advantage from supine positioning in the hammock and thereby provide scientific evidence for its increased use. Further, it is important that neonates are placed supine on a firm mattress in the isolette after being in the hammock before discharge from the neonatal ICU. This practice will reinforce with the parents that the supine sleep position is safe and should always be used because exclusive use of hammocks until the time of discharge may tempt the parents to continue the practice at home.

Furthermore, hammock positioning is one of the methods in complete developmental care, which includes a range 
of interventions to improve the outcomes of neonates, who are constantly under stress in their extrauterine environment. There is controversy in the use of these developmental interventions because they have not consistently shown sustained benefit and there is associated additional cost of care. ${ }^{9}$ This becomes especially more relevant and important for the population at risk for poor developmental outcome such as extreme preterm neonates and those with severe neurologic and respiratory disease when the developmental care is either not given or delayed due to disease-related issues. By identifying clear benefits of hammock use among neonates, the study by Ribas et $\mathrm{al}^{5}$ helps to define the population that should be included in the future studies. The collection of continuous monitoring of outcome data and investigation of long-term outcomes, may together demonstrate sustained neurodevelopmental improvement while simultaneously gaining expertise in providing developmental care when using hammocks in the neonatal ICU, particularly for the more at-risk population.

Shantanu Rastogi

Associate Professor of Clinical Pediatrics SUNY-HSC at Brooklyn Maimonides Medical Center Brooklyn, New York

\section{REFERENCES}

1. Leonel PS, Silva LJ, Porto FR, Santos IMM, Adegas ECV, Gomes $\mathrm{TO}$, et al. The use of the hammock for premature positioning in neonatal ICU: analysis of electronic reports. Rev Fund Care Online 2018;10(1):106-112.

2. Campos Navarro R. Use of hammocks in rural hospital. World Health Forum 19(4)98;19;431-433.

3. Costa KSF, Beleza LO, Souza LM, Ribeiro LM. Hammock position and nesting: comparison of physiological and behavioral effects in preterm infants. Rev Gaucha Enferm 2016;37(spe):e62554.

4. Rodrigues de Jesus V, de Oliveria PMN, Azevedo VMG. Effects of hammock positioning in behavior states, vital signs and pain in preterm: a case series study. Brazil J Phys Therap 2018;22(4):304-309.

5. Ribas C, Adreazza M, Neves V, Silvia V. The effectiveness of hammock positioning in reducing pain and improving sleep wakefulness state in preterm infants. Resp Care 2019.

6. Sahni R. Continuous noninvasive monitoring in the neonatal ICU. Curr Opin Pediatr 2017;29(2):141-148.

7. CPSC, Hamacas Recall Baby Hammocks. Available at https://www. cpsc.gov/Recalls/2009/infant-suffocation-deaths-prompt-recall-ofamby-baby-motion-bedshammocks. Accessed February 12, 2019.

8. Task Force on Sudden Infant Death Syndrome. SIDS and other sleep-related infant deaths: updated 2016. Recommendations for a safe infant sleeping environment. Pediatrics 2016;138(5). pii: e20162938.

9. Symington AJ, Pinelli J. Developmental care for promoting development and preventing morbidity in preterm infants. Cochrane Database Syst Rev 2006;(2):CD001814. 